\title{
Gobierno Electrónico en Colombia: marco normativo y evaluación de tres índices estratégicos*
}

\author{
Cardona Madariaga, Diego Fernando** \\ Cortés Sánchez, Julián David*** \\ Ujueta, Sandra Liliana****
}

\section{Resumen}

Se presenta una revisión del marco normativo sobre modernización del Estado y Gobierno Electrónico en Colombia desde 1995 hasta la fecha, y la evaluación de su despliegue bajo criterios internacionales empleando tres índices durante el período 2003-2012: a) El Índice de Gobierno Electrónico de la Organización de las Naciones Unidas; b) El Networked Readiness Index del Foro Económico Mundial; y c) El Índice de Percepción de Corrupción de Transparencia Internacional. Para realizar un análisis de tendencias de estos índices, se empleó regresión lineal, posiciones relativas y cuartiles. Los resultados permiten evidenciar una constante evolución del marco normativo. La evaluación sobre los índices no es concluyente. Si bien Colombia tiene positivas calificaciones en stock de Capital Humano y alta presencia Web por parte del Gobierno, es esencial avanzar en infraestructura tecnológica y trabajar alrededor de la percepción de corrupción por parte de la ciudadanía.

Palabras clave: Gobierno Electrónico, tecnologías de la información y las comunicaciones, Colombia.

Recibido: 15-11-12 Aceptado: 20-11-14

* Se agradece a Colciencias por la financiación del estudio a través del Programa Nacional de Jóvenes Investigadores-Virginia Gutiérrez de Pineda.

** Profesor Titular de Carrera y Director del Doctorado en Ciencias de la Dirección-Escuela de Administración, Universidad del Rosario, Bogotá, Colombia. E-mail: diego.cardona@urosario.edu.co

*** Profesor Auxiliar de Carrera. Escuela de Administración, Universidad del Rosario, Bogotá, Colombia. E-mail: julian.cortess@urosario.edu.co

**** Coordinadora de Articulación y Acompañamiento para Gobierno en Línea-Ministerio TIC, Colombia. E-mail: sandra.ujueta@gobiernoenlinea.gov.co 


\title{
Electronic Government in Colombia: A Regulatory Framework and the Assessment of Three Strategic Indexes
}

\begin{abstract}
This study presents a review of the regulatory framework dealing with modernization of the State and Electronic Government in Colombia, from 1995 until the present, and assessment of its development according to international criteria, using three indexes for the 2003-2013 period: a) The Index of Electronic Government from the United Nations; b) the Networked Readiness Index from the World Economic Forum; and c) The Index of Perception of Corruption and International Transparency. To analyze the tendencies in these indexes, linear regression, relative and quartile positions were employed. Results made it possible to show a constant evolution of the regulatory framework. Assessment of the indexes is not conclusive. Although Colombia has positive ratings in terms of human capital stock and a high Web presence by the Government, it is essential to advance in technological infrastructure and work around the perception of corruption by the citizenry.
\end{abstract}

Key words: Electronic government, information and communication technologies, Colombia.

\section{Introducción}

El crecimiento exponencial de las Tecnologías de la Información y las Comunicaciones $(\mathrm{TIC})^{1}$ y su adopción so$\mathrm{cial}^{2}$, han tomado importancia en las esferas de la política privada y púbica a nivel internacional ${ }^{3}$ en la llamada sociedad del conocimiento (Drucker, 1969; Kurzweil, 2006; Moore, 1975).

En este escenario, es responsabilidad de los gobiernos construir políticas y marcos normativos que sostengan la incorporación de la sociedad en dinámicas globales y sus cambios tecnológicos (Lara et al, 2013). La innovación de la ad-

1 Entre varias de sus definiciones, las TIC pueden ser comprendidas como: "la sinergia entre hardware, software, redes y medios para la organización, el almacenamiento, procesamiento, transmisión y presentación de información en donde se incluyen también cualquier tipo de dispositivos relacionados para tales fines" (Banco Mundial, 2012a). Vale señalar que las siglas TIC en español es un acrónimo "son" por lo que no modifican su forma cuando designan más de un referente, por lo que no se indica como TIC's.

2 El número total usuarios de Internet a nivel mundial es de 2.267.233.742 (cifras de finales del 2011), llegando a un porcentaje de penetración del 32,7\% (Internet World Stats, 2011). Como segundo ejemplo, el número de suscriptores de líneas celulares en 2011 fue de 5.981.000.000, aproximadamente el $85 \%$ de la población mundial (International Telecommunications Union ITU, 2012).

3 El octavo Objetivo de Desarrollo del Milenio (ODM), específicamente la meta D, aboga por hacer más accesibles los beneficios de las nuevas tecnologías y las TIC a fin de consolidar una alianza mundial para el desarrollo (ONU, 2010). 
ministración pública, está directamente relacionada con su interacción con las TIC y tendrá un impacto exitoso en la medida en que los gobiernos implementen mejoramiento constante y consolide su interacción con actores internos y externos (Criado y Gil, 2013).

De igual manera, es fundamental que los gobiernos implementen las TIC para promover sistemas eficientes para la provisión de servicios públicos a la ciudadanía a través medios digitales. Esta interacción entre las TIC, el sector público y privado, y la sociedad civil, es generalmente conocida como Gobierno Electrónico (GE) (United Nations - UN, 2014).

Dentro de este marco de acción, este estudio tiene el objetivo de analizar la evolución del marco normativo y de las variables relacionadas con GE y modernización del Estado en Colombia desde 1995 hasta la actualidad. Para esto, se elaborará una revisión del marco normativo en Colombia desde 1995 hasta la fecha, esto con el fin de identificar cambios evolutivos en la normativa. Posteriormente, se presentarán los avances relacionados con la estrategia nacional de Gobierno en Línea, esto con el fin de conocer sus adelantos en las fases de adopción. Por último, se evaluará el desempeño de Colombia en variables relacionadas con GE. Se consideró como pertinente explorar las calificaciones alcanzadas por el país frente al examen de organismos internacionales. Para esto, se consultarán tres fuentes: i) Los informes sobre el índice de GE de la Organización de las $\mathrm{Na}$ ciones Unidas (ONU); ii) Los informes del Networked Readiness Index del Foro Económico Mundial (FEM); y iii) Los informes sobre el Índice de Percepción de Co- rrupción de Transparencia Internacional (TI). De cada uno de estos informes, se extraerá como insumo los índices correspondientes. En segunda instancia, para evaluar las tendencias y la evolución del desempeño de Colombia en estos tres frentes durante el período 2003-2012, se empleó una metodología cuantitativa, empleando regresión lineal, posiciones relativas y cuartiles.

\section{Gobierno Electrónico}

Haciendo un símil con la Ley de Moore (1975), los constantes cambios de las aplicaciones de las TIC en el Gobierno impiden un consenso alrededor del concepto de GE (Robertson y Vatrapu, 2012). Para sugerir un punto de partida, se propone que el GE hace referencia al uso de las TIC por parte de las entidades gubernamentales con el fin de brindar servicios públicos y facilitar operaciones que beneficien a los ciudadanos, el sector privado, los servidores públicos y otros grupos de interés (Srivastava y Teo, 2005; Teo et al, 2008; Yang et al, 2014). Esta aplicación tiene la capacidad de transformar las relaciones de actores, entendiendo que su rol en la sociedad es multidimensional, a saber: clientes, sujetos de Estado, "Citoyen" -galicismo que enmarca al ciudadano como participante en la vida política de su comunidad- y votantes (Instituto Nacional de Administración Pública, 2008; Zheng et al, 2014).

EI GE puede servir a una variedad de fines, como (Banco Mundial 2012b; Anderson y Cho 2010): a) Una mejor prestación de los servicios públicos a los ciudadanos; b) Mejor interacción con las empresas y la industria; c) El empodera- 
miento ciudadano a través del acceso a la información; y d) La gestión de un gobierno más eficiente. Los beneficios resultantes pueden ser (Banco Mundial 2012b; Anderson y Cho 2010): a) Menor corrupción; b) Mayor transparencia; c) Mayor comodidad; d) Mayor seguridad; e) Crecimiento en la recaudación de impuestos; $y$, f) Reducción de costos.

El funcionamiento sistémico de los anteriores componentes, se traduce en innovaciones en diversos mecanismos bilaterales de interacción, a saber (Ereminate, 2013; Al-Mobaideen, et al, 2013): 1) G2G: relaciones entre agencias gubernamentales; 2) G2B: relaciones entre las agencias gubernamentales y el sector privado; y 3) G2C: relaciones entre las agencias gubernamentales y el ciudadano.

En síntesis, el GE es un medio por el cual el Gobierno, el sector privado y la sociedad civil, encuentran un espacio viabilizado por las TIC para lograr mayor eficiencia en el uso de recursos, mejorar sus relaciones; $y$, en sumatoria, incrementar las condiciones de bienestar.

\section{Marco normativo del Gobierno Electrónico en Colombia}

Como respuesta a las tendencias regionales e internacionales relacionadas con la Nueva Gestión Pública (NGP) y el GE, el Gobierno colombiano empren- dió una estrategia de modernización del Estado en 1995, con la emisión del documento del Consejo Nacional de Política Económica y Social (CONPES) 2790 titulado: "Gestión Pública Orientada a los Resultados" (Ministerio de TIC, sf ${ }^{4}$ ). En este documento, el Gobierno asume como responsabilidad el uso eficiente y eficaz de los recursos públicos, a fin de movilizar sus actividades hacia un enfoque de interlocución y servicio al ciudadano como eje del desempeño, además de consolidar una gestión pública orientada a los resultados dentro del marco del Plan Nacional de Desarrollo. Sus objetivos específicos consistían en: a) Institucionalizar el concepto de Gestión Integral con Participación Ciudadana; y b) Desarrollar en las entidades públicas la capacidad institucional requerida para cumplir con los objetivos de los programas, subprogramas y proyectos bajo su responsabilidad (Departamento Nacional de Planeación - DNP, 1995).

Luego, con la expedición del Decreto Ley 2150 de 1995, denominado: Decreto Ley Anti trámites del Ministerio de Justicia y Derecho, se especifica en el artículo 26 la obligación del gobierno de habilitar sistemas de transmisión electrónica de datos para que los usuarios envíen o reciban información requerida en sus actuaciones frente a la administración. Esto resulta en un imperativo desde y hacia las entidades públicas para modernizarse. Fue la primera mención del 
uso de medios electrónicos para la realización de trámites (Ministerio de Justicia y Derecho, 1995). Cuatro años más tarde, mediante la Ley 527 de 1999, se define y reglamenta el acceso y uso de los mensajes de datos del comercio electrónico y las firmas digitales, además se establecen las entidades de certificación de firmas digitales, la emisión de certificados electrónicos y la conservación de mensajes de datos (Congreso de Colombia, 1999).

Posteriormente, el documento CONPES 3072 de 2000: "Agenda de Conectividad", se consolida como política que busca: "[M]masificar el uso de las TIC y así aumentar la competitividad del sector productivo, modernizar las instituciones públicas y socializar el acceso a la información" (DNP, 2000: 3). Dentro de sus estrategias, el Gobierno buscaba promover la oferta de información y de servicios a través de la Internet, facilitando la gestión en línea de las entidades gubernamentales e implementando la prestación de servicios públicos mediante el uso de medios electrónicos. De igual forma, busca fortalecer el control social sobre la gestión pública y su función como ofertante de servicios públicos al ciudadano a través del uso de las TIC.
Como plan de acción para el CONPES 3072 de 2000, se expidió la Directiva Presidencial 02 de 2000, para incrementar la eficiencia y la transparen$\mathrm{cia}^{5}$ del Gobierno a través de tres fases ${ }^{6}$ (Presidencia de la República, 2000). También se creó el primer manual del programa de Gobierno en Línea y comenzó un proceso de estandarización de contenidos de los portales Web en las entidades públicas de orden nacional. Adicionalmente, con el Decreto 127 de 2001 se instauró el Programa Presidencial para el Desarrollo de las TIC, el cual coordinaba la Agenda de Conectividad asignándole funciones relacionadas con GE. Sin embargo, más adelante el Decreto 3107 de 2003 reasigna estas funciones al Ministerio de Comunicaciones (Presidencia de la República, 2001; 2003a).

Mediante la Ley 790 del 2002, se impulsa la estrategia nacional de Gobierno en Línea, promoviendo la integración de la información para la gestión pública. La Ley busca ampliar la generación, integración y transparencia de la información en la gestión pública a través del Programa de Reforma a la Administración Pública (PRAP). Adicionalmente impulsa la creación del Sistema Electrónico para la Contratación Pública (SECOP), y la Intra-

5 De manera general, se puede decir que la e-Transparencia hace referencia al uso de las TIC para administrar total o parcialmente hacia la sociedad civil los flujos de información relacionados con el Gobierno y su gestión (Grimmelikhuijsen, et al, 2013; Kim y Lee, 2012; Institute for Development Policy and Management - IDPM, 2012).

6 Fase 1: proveer información en línea a los ciudadanos por parte de todas las entidades involucradas en el programa de Gobierno en Línea. Fase 2: ofrecer servicios y trámites en línea a los ciudadanos. Fase 3: implementar la contratación en línea (Presidencia de la República, 2000). Otros estudios han efectuado un análisis más profundo sobre los progresos de la "Agenda de Conectividad"; véase a Cardona (2006). 
net Gubernamental (Congreso de Colombia, 2002).

Posteriormente, el documento CONPES 3248 de 2003 le da un enfoque territorial a la estrategia de Gobierno en Línea. En ese entonces, 496 entidades contaban con portales en Internet. El diagnóstico elaborado en el documento, identificaba restricciones relacionadas con: a) La falta de responsabilidades sobre la obtención, administración y acceso a la información estratégica para la toma de decisiones; b) Deficiencia en la calidad de la información; c) Incompatibilidad tecnológica de los sistemas implementados; d) Existencia de vacíos regulatorios; y e) Baja capacidad institucional de integración de la tecnología en los procesos regulares de las entidades.

Adicionalmente se menciona que la inversión en sistemas de información de 1996 a 2002 fue de USD\$ 1.222.605.500 a precios constantes del 2001, cuya mayor inversión fue en 1999 con un $28,1 \%$ de este monto, correspondiente a USD $\$ 343.552 .000$ a precios constantes del 2001. La entidad pública que recibió más recursos para la implementación de sistemas de información fue el Departamento Administrativo Nacional de Estadística (DANE) con USD\$ 26.184.500 a precios constantes del 2001. La que recibió menos recursos para la implementación fue el Instituto de Hidrología, Meteorología y Estudios Ambientales (IDEAM) con USD $\$ 26.184 .500$ a precios constantes del 2001 (DNP, 2003).

Luego, mediante el Decreto 3816 de 2003, se articula una estrategia gubernamental con el objetivo de integrar y direccionar las acciones de las entidades públicas generadoras de información. Creando así la Comisión Intersectorial de Políticas y de Gestión de la Información para la Administración Pública. Ésta debía cumplir con el papel de alinear las estrategias y los programas de producción de información, generando espacios para un control social, mejorando las relaciones intragubernamentales con la sociedad civil y sus proveedores, además de optimizar las inversiones del Gobierno (Presidencia de la República, 2003b).

Con la formulación del CONPES 3292 de 2004, en donde se plantea el Proyecto de Racionalización y Automatización de Trámites, se definen tres componentes a solucionar: a) La coordinación interinstitucional y adecuación normativa; b) El análisis funcional para la racionalización; y c) El fortalecimiento tecnológico. También determinó que las entidades deben realizar la simplificación, integración, estandarización y normalización de sus trámites, estableciendo así un plan de acción que busca la racionalización y la automatización (CONPES, 2004).

Las estrategias del CONPES 3292 de 2004, se vieron fortalecidas con la Ley 962 de 2005, en donde se dictan disposiciones sobre racionalización de trámites y procedimientos administrativos y su oferta a través de medios electrónicos. Se destaca que a partir de la expedición de esta Ley se prohibió a las entidades públicas establecer trámites, requisitos o permisos que no estén autorizados por la Ley o el Sistema Único de Información de Trámites (SUIT). Igualmente se vuelve obligatorio su uso y el del Portal del Estado Colombiano (PEC), los cuales son plataformas que unifican el acceso y divulga- 
ción de información, trámites y servicios del gobierno (Congreso de Colombia, 2005).

En 2007 mediante la Ley 1151, se expide el Plan Nacional de Desarrollo 2006-2010. Allí se determina en el artículo 6, numeral 6.2.2, el papel del GE en las políticas de desarrollo como un actor que promoverá la modernización de los esquemas de rendición de cuentas del sector público, la difusión de información sobre las mismas, además de su gestión y resultados, la gestión de trámites, la prestación de servicios, las compras y la contratación pública (DNP, 2007).
En 2008 se emitió el Decreto 1151, en donde se define la implementación de la estrategia de Gobierno en Línea bajo la dirección del Ministerio de Comunicaciones que incluyen aspectos conceptuales, operativos y cronogramas para la implementación del GE en el país. Es relevante destacar las metas y las fechas otorgadas para su logro en el Decreto en mención, las cuales fueron estipuladas de la siguiente forma (Tabla 1) (Ministerio TIC, 2008).

La Ley 1341 de 2009 denominada "Ley de TIC", estipula la masificación del Gobierno en Línea para incrementar los

Tabla 1

Fases de la estrategia de Gobierno en Línea

Fase

Fase de Información ${ }^{7}$

Fase de Interacción ${ }^{8}$

Fase de Transacción ${ }^{9}$

Fase de Transformación ${ }^{10}$

Fase de e-Democracia ${ }^{11}$

Plazo para entidades del orden Plazo para entidades del orden nacional territorial

1 de junio de 2008

1 de diciembre de 2008

1 de diciembre de 2009

1 de junio de 2010

1 de diciembre de 2010
1 de noviembre de 2008

1 de diciembre de 2009

1 de diciembre de 2010

1 de diciembre de 2011

1 de diciembre de 2012

Fuente: Elaboración propia a partir del Ministerio TIC (2008).

7 Fase inicial en la cual las entidades habilitan sus propios sitios Web para proveer información en línea, junto con esquemas de búsqueda básica.

8 Fase en la cual se habilita la comunicación de dos vías entre entidades, ciudadanos y empresas con las consultas en línea e interacción con los servidores públicos.

9 Fase en la que se efectúan transacciones electrónicas para la obtención de productos y servicios

10 Fase en la cual se realizan cambios en la forma de operar de las entidades para organizar los servicios alrededor de necesidades de ciudadanos y empresas, con ventanillas Únicas Virtuales y mediante el uso de la Intranet Gubernamental.

11 Fase en la cual se incentiva a la ciudadanía a participar de manera activa en la toma de decisiones del Estado y la construcción de políticas públicas a través del uso de las TIC. 
servicios prestados a los ciudadanos a través de las TIC; además de fijar mecanismos, condiciones, plazos términos y prescripciones para su desarrollo en el país y las condiciones en que el acceso a la información será garantizado de manera abierta, ininterrumpida y actualizada para adelantar trámites frente a entidades públicas (Congreso de la República, 2009).

Luego, el CONPES 3650 de 2010, estableció la estrategia de Gobierno en Línea como política a través del proyecto "Implementación y Desarrollo de la Agenda de Conectividad", el cual está enfocado en dos líneas: Servicios de Gobierno en Línea; e Intranet Gubernamental. En el primer enfoque, se busca implementar un modelo que involucre acciones de planeación, acompañamiento y ejecución para promover el desarrollo de las fases de Interacción y Transacción estipuladas en el Decreto 1151 de 2008. El segundo enfoque busca integrar las mejores prácticas de operación de infraestructura y soluciones que faciliten la operatividad y el funcionamiento del programa de Gobierno en Línea a través del mejoramiento de la Red de Alta Velocidad del Estado Colombiano (RAVEC), el Centro de Datos y el Centro de Contacto Ciudadano (CONPES, 2010a).

A finales de 2010, el Ministerio TIC presentó el "Plan Vive Digital": una estrategia nacional que busca masificar la pe- netración y la apropiación de Internet. Los resultados fueron: 27 propuestas y 6 temas foco de discusión y participación de las entidades públicas, el sector privado y la sociedad civil. Además, 5.681 personas y entidades registradas aportaron 2.023 sugerencias e iniciativas a través del sitio Web del Ministerio TIC, medios de comunicación y redes sociales. El plan busca alcanzar tres objetivos en cuatro años: 1) Triplicar el número de municipios conectados a la autopista de la información; 2) Conectar a Internet al $50 \%$ de las MIPYMES y al $50 \%$ de los hogares; y 3) Multiplicar por cuatro el número de conexiones a Internet $^{12}$ (Ministerio TIC, 2010).

En 2011, mediante la Ley 1437, todos los órganos del gobierno son incentivados para impulsar el uso de las TIC con el fin de que sus procedimientos se adelanten de manera transparente y sin dilaciones. Adicionalmente, se fortalece el uso de las TIC para la difusión masiva de sus actos, contratos y resoluciones (Congreso de Colombia, 2011a). La Ley 1450 de 2011 por la cual se expidió el Plan Nacional de Desarrollo 2010-2014, presenta en su artículo 227, la obligación a las entidades del gobierno a poner a disposición de manera permanente y gratuita la información que producen y administran. De igual manera, se manifiesta el fomento del uso de las TIC para el desarrollo económico del país en todos los sectores; en

12 El estado actual de los objetivos es el siguiente: 200 municipios conectados a la red de fibra óptica nacional, los cuales se buscan aumentar a $700 ; 7 \%$ de las MIPYMES y $27 \%$ de los hogares conectados a Internet, que se buscan aumentar a 50\% cada uno; y 2,2 millones de conexiones a Internet, las cuales se buscan aumentar a 8,8 millones (Ministerio TIC, 2010). 
los sistemas de información judicial y Justicia Electrónica; además se asegura la continuidad del programa de Gobierno en Línea buscando herramientas y creando servicios que lleguen a todo el territorio nacional (Congreso de Colombia, 2011b; DNP, 2010b).

Finalmente, en el Decreto 19 de 2012, se dictaron normas para reformar procedimientos innecesarios en la Administración Pública. En su artículo 38, se hace mención a la necesidad de mejorar el funcionamiento interno de las entidades públicas que cumplan función administrativa, incrementando la eficiencia y la eficacia en sus procesos mediante el uso de las TIC. De igual forma, se solicita al Ministerio TIC y al programa de Gobierno en Línea apoyo al desarrollo tecnológico para la consulta de información Censal de las comunidades y autoridades indígenas (Presidencia de la República, 2012).

Hasta la fecha de elaboración de este estudio, el programa de Gobierno en Línea está trabajando en un nuevo decreto y el ajuste del "Manual 3.0 para la Implementación de la Estrategia de Gobierno en Línea de las Entidades del Orden Nacional de La República de Colombia" (Ministerio TIC, 2011a). Este nuevo manual, denominado "Manual 3.1", busca: "Incorporar el Gobierno en Línea como parte de la cultura y de la estrategia de innovación organizacional. Conocer las necesidades, tendencias de comportamiento y preferencias de los diferentes grupos de usuarios y aplicar este conocimiento a todos los momentos de interacción con éstos, a la vez que se definen e implementan procesos de mejoramiento permanente en la gestión de tecnologías y en la aplicación de un Sistema de Gestión de Seguridad para la protección de los activos de información" (Ministerio TIC, 2012: 5).

Además de este marco normativo, el Gobierno colombiano ha diseñado y desplegado estrategias para la incidencia directa sobre el desarrollo socioeconómico haciendo uso de las TIC. Dentro del Plan Vive Digital, el Ministerio TIC está implementado los Convenios Regionales de los Fondos para las Tecnologías de la Información y las Telecomunicaciones (FONTIC), los cuales tienen como objetivo proveer conexión a 1.078 municipios través de fibra óptica, permitiendo la provisión de servicios TIC, incrementando el acceso y el servicio universal. A esta iniciativa se suman: "Computadores para Educar" cuyo propósito es masificar el acceso a computadores e Internet en colegios a nivel nacional. Además, se ha avanzado en aspectos tributarios, eximiendo de aranceles e Impuesto al Valor Agregado (IVA) la importación de computadores. Adicionalmente, iniciativas del programa de Gobierno en Línea, como: e-Justicia; Notarías en Línea; Control en Línea y Corporaciones en Línea, están orientadas a fortalecer la transparencia en el Gobierno.

En síntesis, el marco normativo de GE colombiano ha mostrado una evolución sustancial desde el comienzo de las iniciativas de Modernización del Estado, esto con el fin de ajustarse a las dinámicas internacionales de tecnológico en Gobierno sin olvidar el contexto de las necesidades de país. En la próxima sección, se profundizará en los avances de la estrategia nacional de Gobierno en Línea. 


\section{Avances de la estrategia nacional de Gobierno en Línea}

Retomando las fases y fechas estipuladas en el Decreto 1151 de 2008, a continuación se muestran los avances por sector del Estado en cada una de las fases de la estrategia nacional de Gobierno en Línea hasta 2011 (Tabla 2) (CONPES, 2010; Ministerio TIC, 2011b).

A partir de los avances expuestos, se infieren las siguientes observaciones (Tablas 3 y 4 ).

El $44 \%$ de las entidades refleja un avance alto en materia de GE. El $78 \%$ de las entidades evidencia un avance alto en la fase de información. El $56 \%$ de las entidades muestra un avance alto en la fase de interacción. El $67 \%$ de las entidades refleja un avance alto en la fase de transacción. El 30\% de entidades expresa un avance alto en la fase de transformación; $y$ finalmente, el $37 \%$ muestra un avance alto en la fase de e-Democracia.

A nivel nacional, los sectores que presentan un alto nivel de avance en la implementación de GE son: Educación, Economía Solidaria, Estadística, Agricultura y Desarrollo Rural, Ambiente, Vivienda y Desarrollo Territorial, Comercio Industria y Turismo, y TIC. El $78 \%$ de las entidades del orden nacional están cumpliendo con la estrategia de Gobierno en Línea en cada una de las fases en la siguiente proporción: Información: 89\%; Interacción: 85\%; Transacción: 78\%; Transformación: $72 \%$ y e-Democracia: $79 \%$. Como se observa los avances han sido sustanciales, pero si se contrastan con los plazos estipulados en el Decreto 1151 de 2008 tanto en las entidades de orden nacional como territorial, existe un lapso de retraso considerable si, por ejemplo, se tiene en cuenta que la estrategia de Gobierno en Línea debía estar en la fase de e-Democracia el 1ro de diciembre de 2010.

Con relación al capital humano público entrenado, la Tabla 5 presenta las cifras correspondientes al número acumulado entre 2005-2011 de servidores públicos capacitados en la estrategia nacional de Gobierno en Línea e Intranet Gubernamental (Ministerio TIC, 2011c).

En síntesis, la estrategia nacional de Gobierno en Línea ha consolidado avances en cada uno de los sectores mediante la evolución en cada una de sus fases: información, interacción, transacción, transformación y democracia. Sin desmeritar estos avances, igualmente, se identifican a su vez retrasos perjudiciales tanto en entidades de orden territorial y nacional.

En la siguiente sección, se presenta el análisis de tres índices estratégicos relacionados con GE con la finalidad de contrastas la evolución del marco normativo con la calificación otorgada al país por parte de organismos internacionales: ONU, Foro Económico Mundial y Transparencia Internacional.

\section{Análisis comparativo de índices estratégicos}

En esta sección, se comparará el desempeño que ha tenido Colombia en los escalafones de tres índices estratégicos, a saber: Índice de GE (IGE) de la ONU; Networked Readiness Index (NRI) del Foro Económico Mundial (FEM); y el 


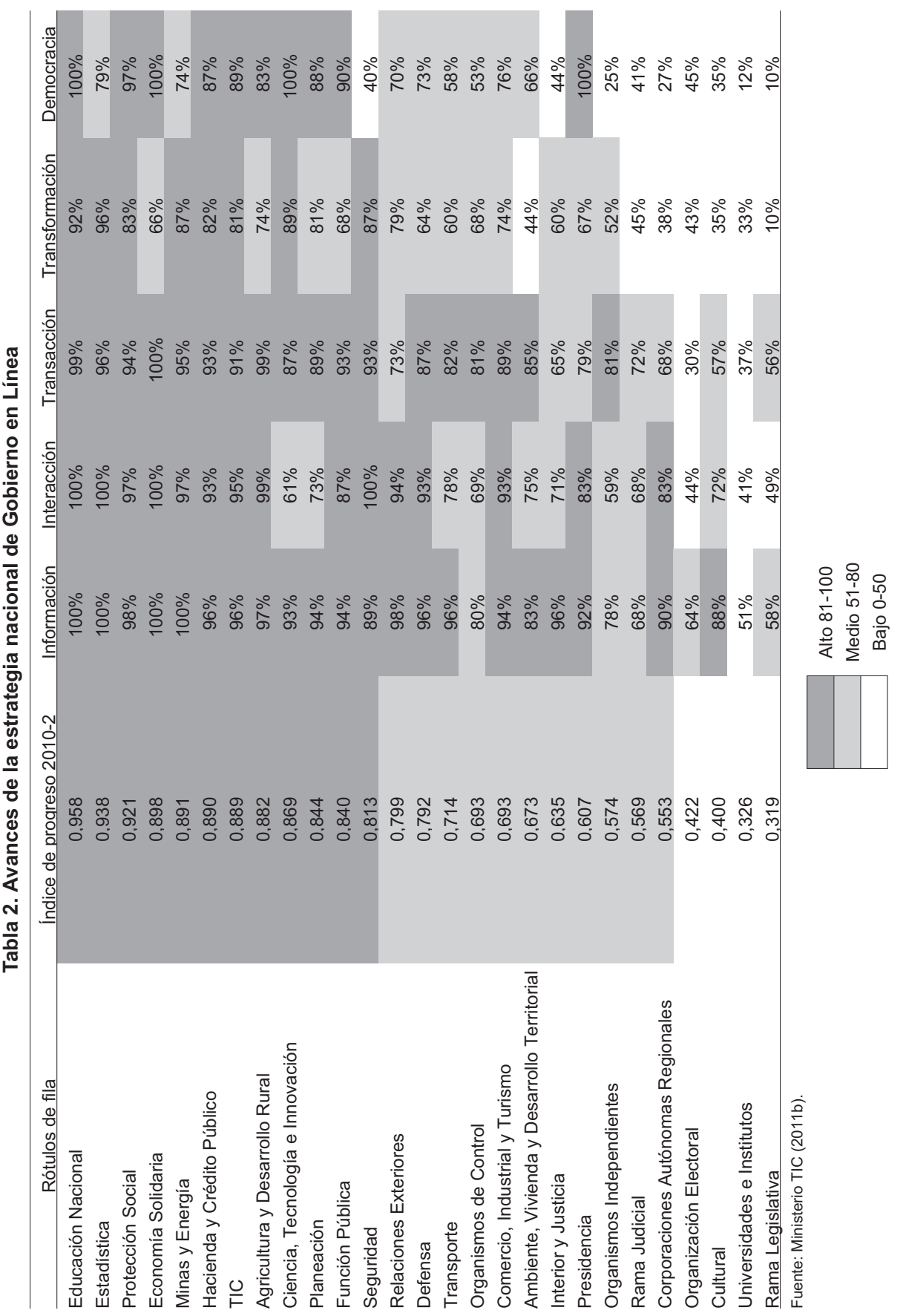


Tabla 3

Índice de avance de ramas, sectores o entidades en el programa de Gobierno en Línea

\begin{tabular}{lrr}
\hline \multicolumn{1}{c}{ Índice } & $\#$ & $\%$ \\
\hline Alto & 12 & $44 \%$ \\
Medio & 11 & $41 \%$ \\
Bajo & 4 & $15 \%$ \\
Total de ramas, sectores, & 27 & \\
entidades & & \\
\hline
\end{tabular}

Fuente: Elaboración propia a partir del Ministerio TIC (2011b).

Índice de Percepción de Corrupción (IPC) de la Organización Transparencia Internacional (TI).

De manera introductoria, se examinarán los componentes que estructuran los índices mencionados. Luego se analizarán las tendencias de los índices a través de un análisis de regresión lineal sim- ple, tomando como variables independientes los años de los informes y como variables dependientes la calificación de Colombia en cada uno de los índices. Al desarrollar la relación entre estas dos variables se derivará una recta, de la cual se podrá inferir su pendiente y el coeficiente de determinación o R2, lo cual servirá para: a) Saber si la tendencia del desempeño del país es ascendente o descendente y en qué magnitud; $y$ b) La bondad de ajuste de la regresión o, en otras palabras, el porcentaje de la variación de las calificaciones que se puede explicar con la regresión entre los años evaluados y la calificación de Colombia (Anderson et al, 1999).

Posteriormente se realizará una comparación entre el IGE, el NRI y el IPC a través de posiciones relativas y cuartiles, a fin de visualizar el comportamiento

Tabla 4

Índice de avance de ramas, sectores o entidades en el programa de Gobierno en Línea por número, porcentaje y fases del Decreto 1151 de 2008

\begin{tabular}{|c|c|c|c|c|c|c|c|c|c|c|}
\hline Fases & Info & ación & Int & ción & Tral & cción & Trans & nación & Der & racia \\
\hline Avance & $\mathrm{N}^{\circ}$ & $\%$ & $\mathrm{~N}^{\circ}$ & $\%$ & $\mathrm{~N}^{\circ}$ & $\%$ & $\mathrm{~N}^{\circ}$ & $\%$ & $\mathrm{~N}^{\circ}$ & $\%$ \\
\hline Alto & 21 & $78 \%$ & 15 & $56 \%$ & 18 & $67 \%$ & 8 & $30 \%$ & 10 & $37 \%$ \\
\hline Medio & 5 & $19 \%$ & 9 & $33 \%$ & 7 & $26 \%$ & 12 & $44 \%$ & 8 & $30 \%$ \\
\hline Bajo & 1 & $4 \%$ & 3 & $11 \%$ & 2 & $7 \%$ & 7 & $26 \%$ & 9 & $33 \%$ \\
\hline
\end{tabular}

Fuente: Elaboración propia a partir del Ministerio TIC ( 2011b).

\section{Tabla 5}

Número de funcionarios públicos/contratistas capacitados/sensibilizados en Gobierno en Línea/Intranet Gubernamental

\begin{tabular}{ccc}
\hline Componente & Indicador/Estadística & Acumulado 2005-2011 \\
\hline Servicios de Gobierno en Línea & $\begin{array}{c}\text { Número de servidores públicos y } \\
\text { contratistas del orden territorial } \\
\text { sensibilizados y capacitados }\end{array}$ & 81,426 \\
Intranet Gubernamental & $\begin{array}{c}\text { Número de servidores públicos y } \\
\text { contratistas capacitados }\end{array}$ & 10,274 \\
\hline
\end{tabular}

Fuente: Elaboración propia a partir del Ministerio TIC (2011). 
de Colombia en función del número de países evaluados en cada escalafón en un rango de posiciones estándar. Se empleará como elemento comparativo el desempeño que ha tenido Colombia en el escalafón del IGE en los años: 2003, $2004,2005,2008,2010$ y 2012, años en los cuales se publicó informe. Si bien los otros dos índices proveen información en los años donde no se realizaron estudios sobre el IGE, no se tendrán en cuenta por la ausencia de información para su comparación.

\section{1. Índice de Gobierno Electrónico de la Organización de las Naciones Unidas}

El imperativo de los estudios sobre GE a nivel mundial, inició debido a la consideración por parte de los Gobiernos de dos aspectos relacionados con el crecimiento económicos de las naciones: 1) EI rápido avance de la globalización ha convertido el comercio entre países, las inversiones y las oportunidades financieras en redes transaccionales que buscan proporcionar productos y servicios más competitivos; y 2) Los avances en el uso de las TIC han presentado nuevos acercamientos para la integración de redes y el mejoramiento de la eficiencia de los negocios y los servicios a nivel mundial (ONU, 2003).

Por otra parte, el GE se puede concebir como una herramienta para el desarrollo si se cumplen al menos tres prerrequisitos: i) Un umbral mínimo de infraestructura tecnológica; ii) La existencia considerable de capital humano; y iii) Buenos alcances de conectividad (ONU, 2003).
A su vez, las anteriores dos variables tienen relación intrínseca con dos procesos que integran el GE: 1) La capacidad genérica y aptitud del sector público para implementar las TIC en el despliegue de los servicios ofertados y la mediación para bridar información de alta calidad y herramientas de comunicación efectiva que soporten el desarrollo humano; esto es denominado como la disposición al GE (e-Gov Readiness); y 2) La voluntad por parte del Gobierno para utilizar las TIC con el fin de proporcionar información de alta calidad y herramientas de comunicación efectiva para el propósito específico de capacitar a las personas para que sean partícipes en dinámicas de consulta y toma de decisiones, como consumidores de servicios públicos y ciudadanos; esto es denominado como e-Participación (e-Participation) (ONU, 2003).

EI IGE es un indicador compuesto por tres subíndices cuantitativos: i) Subíndice de Medición Web, el cual mide la aptitud de los gobiernos para emplear estrategias de GE como herramientas para informar, interactuar, tramitar e interconectar. Está basado en un modelo teórico de cinco fases denominado Web Presence Measurement Model. Las cinco fases son: presencia emergente, presencia mejorada, presencia interactiva, presencia transaccional y presencia interconectada; ii) Subíndice de Infraestructura Tecnológica: mide el capital tecnológico de un país. Está compuesto por seis subíndices: computadores cada 1000 personas; usuarios de Internet cada 1000 personas; líneas telefónicas cada 1000 personas; población On-Line 
cada 1000 personas; celulares cada 1000 personas y televisores cada 1000 personas; y iii) Subíndice de Capital Humano: índice dependiente del Programa de las Naciones Unidas para el Desarrollo. Es un componente de la tasa de alfabetismo en adultos y su relación combinada con la educación primaria, secundaria y terciaria. Está dividido en dos tercios de importancia a la alfabetización adulta y un tercio a la tasa bruta de matriculación (ONU, 2003).

Por tanto, se puede entender el IGE como un indicador multidimensional que comprende componentes relaciona- dos con el uso de las TIC por parte de la población y su implementación para optimizar múltiples activos del desarrollo como el Capital Humano, la disminución de la Brecha Digital ${ }^{13}$ y la infraestructura tecnológica y de un país.

Para profundizar el análisis sobre el desarrollo que Colombia ha tenido en cada uno de los subíndices mencionados y en el IGE, a continuación se presentan las calificaciones y sus respectivas tendencias durante el período 2003-2012 (Figura 1).

La ecuación de regresión lineal (ERL) para el IGE es $Y=0,0194 x-38,283$

Figura 1

Tendencia del desempeño de los tres subíndices del IGE en Colombia 2003-2012

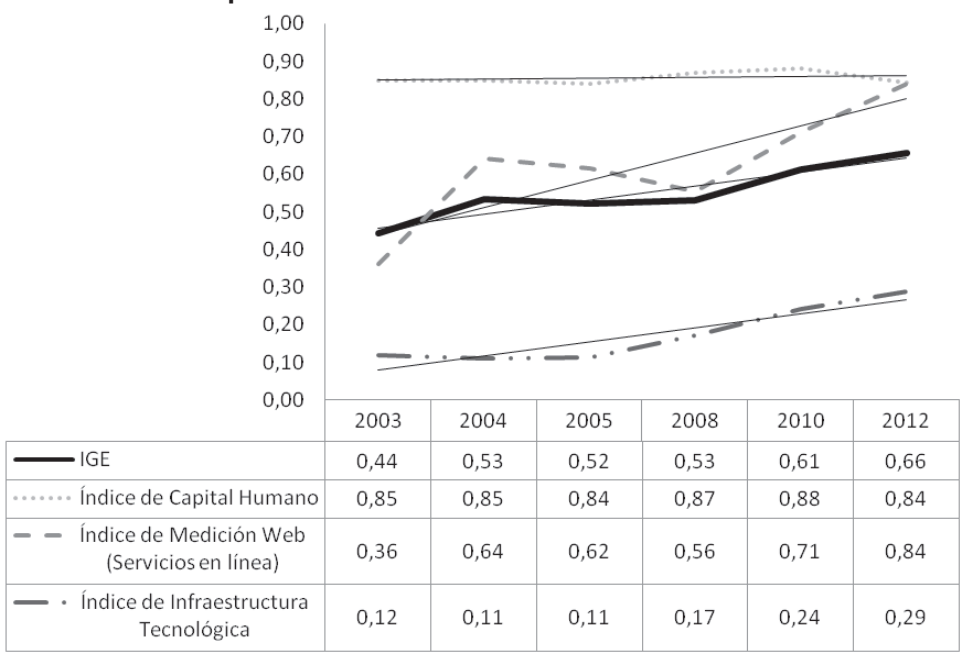

Fuente: Elaboración propia a partir de la ONU 2003-2012.

13 La OECD (2001: 5) hace referencia al término de Brecha Digital como: "la disparidad entre individuos, hogares, negocios y áreas geográficas en diferentes niveles socioeconómicos, con respecto a sus distintos accesos a las TIC y el uso que le dan a la Internet dentro de una variedad de actividades". 
con un $\mathrm{R}^{2}=0,84$. La pendiente positiva indica una tendencia ascendente en el desempeño global del índice. La ERL para el subíndice de capital humano es $Y=0,0015 x-2,2364$ con un $R^{2}=0,1162$. La pendiente positiva indica una tendencia estancada en este factor, no obstante, este subíndice presenta una calificación significativamente mayor que los otros dos. La ERL para el subíndice de medición Web es $Y=0,0357 x-70,957$ con un $R^{2}=0,6383$. La pendiente positiva indica una tendencia ascendente en este factor; en efecto, es el subíndice con mayor pendiente dentro del período analizado del IGE. La ERL para el subíndice de infraestructura tecnológica es $Y=0,0206 x-$ 41,259 con un $R^{2}=0,9326$. La pendiente positiva indica una tendencia ascendente en este factor; sin embargo, es el factor con menor calificación dentro de los subíndices del IGE.

El anterior análisis, permite inferir que el desempeño de Colombia en el IGE está reforzado por la decisiva presencia Web del gobierno, con lo cual se posiciona como líder regional en esta materia y en participación en línea. Por otro lado, frente a factores de Capital Humano e Infraestructura Tecnológica, Colombia necesita fortalecer sustancialmente cobertura y calidad educativa, además de disminuir la Brecha Digital.

\subsection{Networked Readiness Index}

El Networked Readiness Index (NRI) del Foro Económico Mundial (FEM), es definido como el grado de preparación de una nación o una comunidad determinada para participar y beneficiar- se del uso de las TIC (FEM, 2003). Este índice separa los factores de entorno, preparación y uso de TIC. Por tanto, hay tres índices principales compuestos por más de 90 variables. Los subíndices y sus principales constituyentes son: i) Índice del entorno, que se divide en subíndice de mercado, subíndice del entorno político y regulatorio y subíndice de infraestructura; ii) Índice del grado de preparación (readiness), que se divide en, subíndice de preparación individual, subíndice que preparación del sector privado y subíndice de preparación del sector público; y iii) Índice de uso de las TIC, que se divide en subíndice de uso de las TIC individual, subíndice uso de las TIC en el sector privado y subíndice de uso de las TIC en el sector público (FEM, 2003).

Para profundizar el análisis sobre el grado de preparación de Colombia en relación al uso y apropiación de las TIC para el desarrollo, a continuación se presenta su calificación y tendencia durante el período 2003-2012 (Figura 2).

La ERL para el NRI es $Y=0,0928 x$ 182,78 con un $R^{2}=0,84$. La pendiente positiva indica una tendencia ascendente en el NRI, de hecho, superando pendiente del subíndice de medición Web. Este desempeño analizado comunica que la planeación y ejecución de las políticas nacionales relacionadas con entorno, preparación y uso de las TIC, son coherentes con las dinámicas privadas y sociales del país en función de su ingreso progresivo a la sociedad de la información y el conocimiento, $y$, adicionalmente, han tenido evaluaciones positivas bajo la lente del FEM. 


\section{3. Índice de Percepción de Corrupción}

A diferencia de la complejidad de la construcción de los anteriores dos índices debido a la cantidad de variables involucradas, su organización e interrelación temática, el Índice de Percepción de Corrupción (IPC) realizado por la organización Transparencia Internacional (TI) es un indicador que refleja la percepción de corrupción en sectores académicos, empresariales y analistas de riesgo ( $\mathrm{Tl}$, 2003). Estos actores deben dar un concepto cuantificable de su percepción de corrupción en el entorno de 1 a 10, siendo
10 la máxima calificación de transparencia. Para profundizar el análisis sobre percepción de corrupción en Colombia a continuación se presenta su calificación y tendencia durante el período 2003-2012 (Figura 3).

La ERL para el IPC es $Y=-0,0297 x+63,316$ con un $R^{2}=0,6337$. La pendiente negativa indica una tendencia descendente en el desempeño de Colombia en el IPC. Esto sugiere que, a pesar de las evaluaciones y resultados positivos que han tenido las estrategias de modernización del estado, GE, participación electrónica y políticas nacionales relacionadas con tecnología, se deben im-

Figura 2

Tendencia del desempeño del NRI para Colombia 2003-2012

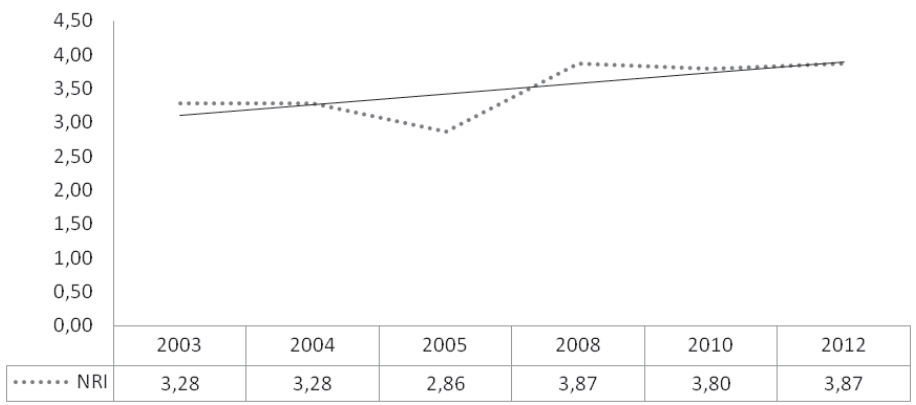

Fuente: Elaboración propia a partir de FEM, 2003-2012.

Figura 3

Tendencia del desempeño del IPC para Colombia 2003-2012

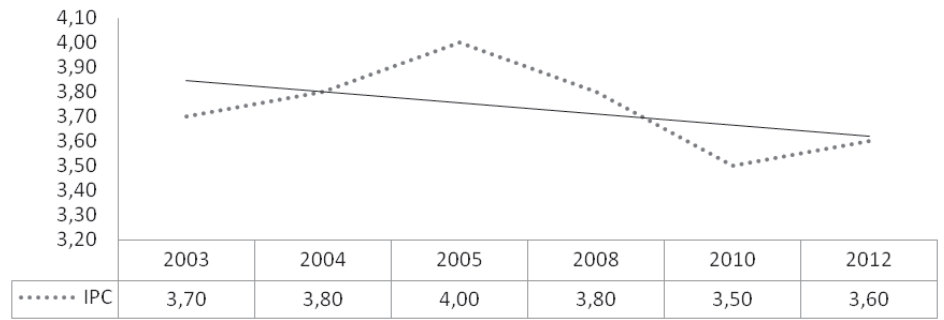

Fuente: Elaboración propia a partir de TI 2003-2012. 
plementar estos factores a modo de apalancamiento para mejorar la percepción de la ciudadanía colombiana con relación al funcionamiento del Gobierno.

Es de relevancia mencionar que si bien los procesos de modernización del Estado son medulares para incrementar la transparencia, hay un álgido debate detrás de esta afirmación. En principio, Michener y Bersch (2013) han propuesto dos dimensiones para conceptualizar la transparencia. La primera dimensión es visibilidad, la cual consiste en el grado de facilidad para encontrar información completa y fácil sobre el sector público. Esta dimensión comprende dos subdimensiones: la transparencia clara y la transparencia opaca. En este plano, Fox (2007; 2008) argumenta que la transparencia clara es un escenario en donde el flujo de información dentro y fuera del Gobierno es libre, masivo y detallado. En cambio, la transparencia opaca es un escenario en donde los procesos de difusión son nominales $\mathrm{y}$, por tanto, hay múltiples restricciones para acceder a información relacionada con el sector público. La segunda dimensión, es la derivabilidad: la magnitud en la que la información sobre el sector público puede ser aprovechada para promover la participación ciudadana en la toma de decisiones.

La inmersión de diferentes gobiernos locales y nacionales en las dimensiones de visibilidad y derivabilidad, es evidenciada en diferentes estudios de caso. Kim y Lee (2012) afirman que las estrategias de GE y transparencia incrementa la percepción de la ciudadanía sobre fu influencia en la toma de decisiones del Gobierno local. Sin embargo, Grimmelikhuijsen et al (2013) encontraron que los efectos de la transparencia no son generalizables y que dependen en gran medida de la cultura local. De una manera más enfática, Roberts (2006) concluye que la transparencia no tiene ningún efecto en la percepción de confianza de la ciudadanía hacia el Gobierno.

Para resumir, si bien el debate sobre la modernización del Estado, el GE y sus estrategias para disminuir la percepción de corrupción de la ciudadanía y otros elementos de su propiedad multidimensional sobrepasa el alcance de este trabajo, se considera un primer avance analizar en paralelo la evolución de su marco normativo en el país y la resultados en la percepción ciudadana consultada por TI a lo largo del período de análisis.

\subsection{Análisis comparativo de índices}

La estructura del análisis comparativo de los índices se presentará desde dos perspectivas complementarias: i) El desempeño de Colombia en función de posiciones relativas correspondientes a cada año ya que en la mayoría de ocasiones el número de países analizados en cada informe es distinto; la posición relativa es la posición de Colombia en determinado año e informe dividida por el número total de países evaluados en el mismo (Figura 4); y ii) El desempeño de Colombia en los escalafones de cada índice partiendo de su ubicación dentro del rango de cuartiles (Qn). Este análisis adicional reforzará las inferencias de las posiciones relativas ya que determinará las fluctuaciones de Colombia en los escalafones para cada año e informe en rangos estandarizados (Tabla 6). 
Figura 4

Evolución conjunta como posiciones relativas del IGE, NRI y el IPC 2003-2012

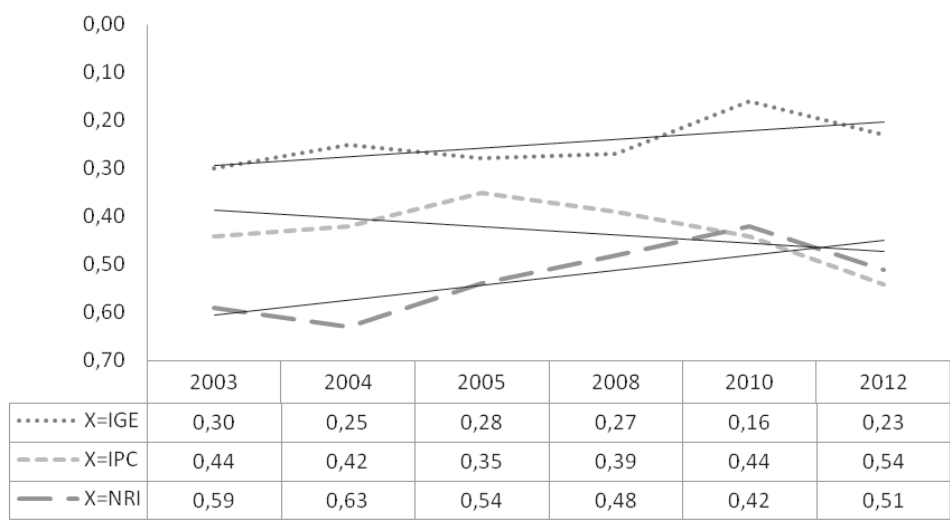

Fuente: Elaboración propia a partir de ONU, 2003-2012; FEM, 2003-2012; TI, 2003-2012.

Tabla 6

Ubicación en cuartiles de Colombia en el IGE-ONU, NRI y IPC

\begin{tabular}{cccccccccc}
\hline \multirow{2}{*}{ Año } & \multicolumn{3}{c}{ IGE-ONU } & \multicolumn{3}{c}{ NRI } & \multicolumn{3}{c}{ IPC } \\
\cline { 2 - 9 } & Posición \# Países & Qn & Posición \# Países & Qn & Posición \# Países & Qn \\
\hline 2003 & 57 & 191 & Q2 & 60 & 102 & Q3 & 59 & 133 & Q2 \\
2004 & 44 & 178 & Q1 & 66 & 104 & Q3 & 60 & 143 & Q2 \\
2005 & 54 & 191 & Q2 & 62 & 115 & Q3 & 55 & 159 & Q2 \\
2008 & 52 & 192 & Q2 & 64 & 134 & Q2 & 70 & 180 & Q2 \\
2010 & 31 & 192 & Q1 & 58 & 138 & Q2 & 78 & 178 & Q2 \\
2012 & 43 & 190 & Q1 & 73 & 142 & Q3 & 94 & 174 & Q3 \\
\hline
\end{tabular}

Fuente: Elaboración propia a partir de ONU, 2003-2012; FEM, 2003-2012; TI. 2003-2012.

La ERL de las posiciones relativas del IGE es $Y=0,0095 x+19,378$ con un $R^{2}$ $=0,4733$; la ERL de las posiciones relativas del NRI es $Y=0,0163 x+33,142$ con un $\mathrm{R}^{2}=0,5892$; y la $E R L$ de las posiciones relativas del IPC es $Y=-0,0108 x+21,244$ con un $\mathrm{R}^{2}=0,365$. A partir de la anterior información se puede inferir que: i) El desempeño de Colombia en el escalafón del IGE presenta una tendencia ascendente inferior al desempeño de sus subíndices internos, no obstante mantiene un posi- cionamiento consistente entre $Q_{1}$ y $Q_{2}$; ii) Lo mismo sucede con relación a ambas tendencias dentro del NRI con la diferencia que en cuatro de los seis años analizados se ha ubicado en el $\mathrm{Q}_{3}$; por último, iii) La tendencia de las posiciones relativas en el IPC es positiva si se compara con su desempeño a partir de las calificaciones; a pesar de que ambas tendencias son negativas, su rendimiento en las posiciones relativas no es descendente de manera precipitada, ocupando cinco de 
los seis años en el $Q_{2}$, aunque en el 2012 terminó en el rango de $\mathrm{Q}_{3}$ (ONU, 20032012; FEM, 2003-2012; TI, 2003-2012).

\section{Conclusiones}

A mediados de los años 90 el Estado colombiano emprendió el camino hacia la modernización siguiendo las tendencias internacionales de la Nueva Gestión Pública. La construcción de un marco normativo para el despliegue de diversas estrategias incluyó leyes, decretos y políticas públicas orientadas al uso eficiente y eficaz de los recursos públicos, a la implementación de sistemas de información para la gestión de información interna y la comunicación con la ciudadanía, y un enfoque territorial consciente del contexto socioeconómico de cada región.

Adicionalmente, el Gobierno también ha llevado a cabo estrategias que inciden de manera más directa en el desarrollo socioeconómico como la masificación de fibra óptica y del servicio de Internet, o la dotación y acompañamiento en el uso y apropiación de herramientas tecnológicas para educación. Si bien es posible identificar un progreso global en el marco normativo y en la política pública, un elemento para considerar es el atraso en el cumplimiento de los plazos definidos en el Decreto 1151 de 2008 para las fases de la estrategia de Gobierno en Línea.

Por otro lado, las evaluaciones elaboradas por la ONU, el FEM y TI sobre variables relacionadas con el GE son mixtas. En el IGE de la ONU, Colombia presenta una tendencia positiva en los escalafones. Cuando se desagregan los subíndices del IGE, el Capital Humano sostiene la calificación más elevada y un comportamiento inercial. En segundo lugar, el país sobresale a nivel regional en presencia Web del Gobierno. No obstante, la infraestructura tecnológica del país no permite una reducción más efectiva de la Brecha Digital.

En el caso del NRI, se observa una tendencia ascendente en la calificación del país. Al contrastar este índice con el IGE, es posible deducir que los factores de políticas públicas y macroeconómicas para el aprovechamiento de las TIC en el crecimiento, están acondicionados para su óptimo despliegue. Lo que apalancaría la posición de Colombia en la sociedad del conocimiento a través de un uso y apropiación más intensiva de las TIC es: Primero, una formación de Capital Humano desde la educación para el uso productivo de estas herramientas; y segundo, la reducción de la Brecha Digital.

En el IPC, la tendencia de Colombia en los escalafones es descendente. Como se mencionó en la sección posterior al análisis de estos resultados, las evidencias sobre el impacto de las estrategias de GE en la transparencia no son unánimes. Mientras el país es evaluado cada vez mejor en el IGE y en el NRI, el IPC no otorga un parte integralmente positivo.

Con todo lo anterior, se puede concluir que Colombia ha avanzado tanto en la normativa como en los índices de evaluación internacional de elementos relacionados con el GE. Independientemente de ello, si bien un porcentaje considerable de las ramas, sectores y entidades del Gobierno han alcanzado indicadores de 
progreso en las fases de la estrategia nacional de Gobierno, su proceso de actualización y mejora sigue en construcción.

El Gobierno necesita fortalecer aspectos de entorno, principalmente: consolidar el cumplimiento de las metas consignadas en su marco normativo; ampliar y fortalecer la infraestructura tecnológica en conjunto con programas de formación y capacitación del Capital Humano para el uso y apropiación de las TIC. De esta manera, puede ampliar la cobertura de las estrategias de GE para la provisión de servicios públicos, y de este modo, ser más eficiente, transparente y permitir la participación ciudadana en la toma de decisiones públicas.

\section{Referencias bibliográficas}

Al-Mobaideen, Hisham, Al-Bashabsheh, Samer, Basioni, Eman y Al-Khawaldeh, Abeer (2013). Electronic government services and benefits in Jordan. International Journal of Academic Research, Vol. 5, No. 6, Azerbaijan, pp. 206-215.

Anderson, David; Sweeney, Dennis y Williams, Thomas (1999). Estadística para Administración y Economía, México, Thomson.

Anderson, Dennis y Cho, June-Suh (2010). "The role of public service in achieving the Millennium Development Goals". Presentado en: United Nations Public Service Day-Awards and Forum. 21 - 23 de junio de 2010. Barcelona, España. Disponible en: http://goo.gl/ HqeYA, recuperado el 2 de julio de 2013.

Banco Mundial - BM (2012a). Glosario de definiciones de Tecnologías de la Información y las Comunicaciones, sitio Web del Banco Mundial: http://goo
.gl/QysOH, recuperado el 4 de mayo de 2013.

Banco Mundial - BM (2012b). Glosario de definiciones de Gobierno Electrónico, sitio Web del Banco Mundial: http://goo.gl/ZrQOI, recuperado el 4 de mayo de 2013.

Cardona, Diego (2006). The Conectivity Agenda: Five years of success in Colombian e-Gov initiatives. Universidad y Empresa, Vol. 5, No. 11, Colombia, Facultad de Administración, Universidad del Rosario, pp. 142-162.

Congreso de Colombia (1999). Ley 527 de 1999. Recuperado el 4 de mayo de 2013, del sitio Web del Observatorio Colombiano de Ciencia y Tecnología: http://goo.gl/0blFJ, Bogotá, Colombia.

Congreso de Colombia (2002). Ley 790 de 2002. Recuperado el 4 de mayo de 2013, del sitio Web de la Secretaría del Senado: http://goo.gl/hpsMW, Bogotá, Colombia.

Congreso de Colombia (2005). Ley 962 de 2005. Recuperado el 4 de mayo de 2013, del sitio Web de la Alcaldía de Bogotá, sistema de consulta de normas: http://goo.gl/ClwUV. Bogotá, Colombia.

Congreso de Colombia (2009). Ley 1341 de 2009. Recuperado el 4 de mayo de 2013, del sitio Web de la Secretaría del Senado: http://goo.gl/v0K90, Bogotá, Colombia.

Congreso de Colombia (2011a). Ley 1437 de 2011. Recuperado el 4 de mayo de 2013, del sitio Web de la Alcaldía de Bogotá, sistema de consulta de normas: http://goo.gl/k89yY, Bogotá, Colombia.

Congreso de Colombia (2011b). Ley 1450 de 2011. Recuperado el 4 de mayo de 2013, del sitio Web de la Secretaría del Senado: http://goo.gl/YdHwi, Bogotá, Colombia. 
Criado, J. Ignacio y Gil, J. Ramón (2013). Electronic government and Public Policy: Current status and future trends, Gestión y Política Pública, México, Centro de Investigación y Docencia Económicas-CIDE, pp. 3-48.

Departamento Nacional de Planeación-DNP (1995). Documento CONPES 2790 de 1995. Recuperado el 4 de mayo de 2013, del sitio Web del Departamento Nacional de Planeación, sistema de búsqueda de documentos: catálogo, bilioteca: http://goo.gl/muF3L, Bogotá, Colombia.

Departamento Nacional de Planeación-DNP (2000). Documento CONPES 3072 de 2000. Recuperado el 4 de mayo de 2013, del sitio Web del Departamento Nacional de Planeación, sistema de búsqueda de documentos: catálogo, bilioteca: http://goo.gl/muF3L, Bogotá, Colombia.

Departamento Nacional de Planeación-DNP (2003). Documento CONPES 3248 de 2003. Recuperado el 4 de mayo de 2013, del sitio Web del Departamento Nacional de Planeación, sistema de búsqueda de documentos: catálogo, bilioteca: http://goo.gl/muF3L, Bogotá, Colombia.

Departamento Nacional de Planeación-DNP (2004). Documento CONPES 3292 de 2004. Recuperado el 4 de mayo de 2013, del sitio Web del Departamento Nacional de Planeación, sistema de búsqueda de documentos: catálogo, bilioteca: http://goo.gl/muF3L, Bogotá, Colombia.

Departamento Nacional de Planeación-DNP (2007). Plan Nacional de Desarrollo 2006-2010. Recuperado el 4 de mayo de 2013, del sitio Web del Departamento Nacional de Planeación, sistema de búsqueda de documentos: catálogo, bilioteca: http://goo.gl/muF3L, Bogotá, Colombia.
Departamento Nacional de Planeación-DNP (2010a). Documento CONPES 3650 de 2010. Recuperado el 4 de mayo de 2013, del sitio Web del Departamento Nacional de Planeación, sistema de búsqueda de documentos: catálogo, biblioteca: http://goo.gl/muF3L, Bogotá, Colombia.

Departamento Nacional de Planeación-DNP (2010b). Plan Nacional de Desarrollo 2010-2014. Recuperado el 4 de mayo de 2013, del sitio Web del Departamento Nacional de Planeación, sistema de búsqueda de documentos: catálogo, biblioteca: http://goo.gl/mu F3L, Bogotá, Colombia.

Drucker, Peter (1969). The Age of Discontinuity, United States, Harper y Row.

Ereminate, Simona (2013). The model of integration of Electronic Government and Electronic Business in Public Procurement. Regional Formation and Development Studies, Vol. 9, No. 1, Lithuania, Klaipeda University, pp. 39-51.

Foro Económico Mundial-FEM (2003). The Networked Readiness Index, sitio Web del Foro Económico Mundial: http://goo.gl/0R9w1, recuperado el 4 de mayo de

Foro Económico Mundial-FEM (2003-2012). The Networked Readiness Index Ranking 2003-2012, sitio Web del Foro Económico Mundial: http://goo. gl/0R9w1, recuperado el 4 de mayo de 2013.

Fox, Jonathan (2008). Transparencia y rendición de cuentas, En J. Ackerman, Más allá del acceso a la información: Transparencia, rendición de cuentas y Estado de derecho (pp. 174-198), Méxi$\mathrm{co}$, Instituto de Investigaciones Jurídicas-UNAM/CETA/Universidad de Guadalajara/Siglo XXI Editores.

Grimmelikhuijsen, Stephan; Porumbescu, Gregory; Hong, Boram, y Im, Tobin 
(2013). The effect of transparency on trust in government: A cross-national comparative experiment, Public Administration Review, Vol. 73, No. 4, United States, American Society for Public Administration, pp. 575-586.

Institute for Development Policy and Management-IDPM (2012). Transparency and e-Transparency Glossary, sitio Web del IDPM: http://goo.gl /QYOWj, recuperado el 4 de mayo de 2013.

Instituto Nacional de Administración Pública (2008). e-Gobierno para un mejor Gobierno, España, OCDE.

International Telecommunication Union - ITU (2012). Key Global Telecommunication Indicators for the World Telecommunication Service Sector, sitio Web de la ITU: http://goo.gl/MTiap, recuperado el 9 de mayo de 2013.

Internet World Stats (2011). Internet World Stats, sitio Web Internet World Stats: http://www.internetworldstats.com/, recuperado el 4 de mayo de 2013.

Kim, Soonhee y Lee, Jooho (2012). EParticipation, Transparency and Trust in Local Government. Public Administration Review, Vol. 72, No. 6, United States, pp. 819-828.

Kurzweil, Raymond (2006). The Singularity Is Near: When Humans Transcend Biology, London, Penguin Books.

Lara, Marco; Martínez, Vicente; y Torres, Lourdes (2013). Electronic Government and Accountability in Regional and State Administration. Gestión y Política Pública, México, Centro de Investigación y Docencia Económicas-CIDE, pp. 105-135.

Michener, Greg y Bersch, Kate (2013). Identifying transparency, Information Policy, Vol. 18, United States, Institute for Information Policy-The Pennsylvania State University, pp. 233-242.

Ministerio de Justicia y Derecho (1995). Decreto 2150 de 1995 , recuperado el 9 de mayo de 2013, del sitio Web de la Alcaldía de Bogotá, sistema de consulta de normas: http://goo.gl/yW2uO. Bogotá, Colombia.

Ministerio de las Tecnologías de la Información y las Comunicaciones - Ministerio TIC (s.f.). Normativa del Gobierno Electrónico en Colombia: Programa Gobierno en Línea, sitio Web: Programa del antiguo plan de Gobierno en Línea: http://goo.gl/cPSmd, recuperado el 9 de mayo de 2013.

Ministerio TIC (2008). Decreto 1151 de 2008, recuperado el 9 de mayo de 2013, del sitio Web del Programa de Gobierno en Línea: http://goo.gl/bCRHo. Bogotá, Colombia.

Ministerio TIC (2010). Plan Vive Digital, sitio Web del Ministerio TIC: http://www. mintic.gov.co/index.php/vive-digital /plan/objetivos-aspiraciones, recuperado el 9 de mayo de 2013.

Ministerio TIC (2011a). Manual 3.0 para la Implementación de la Estrategia de Gobierno en Línea de las Entidades del Orden Nacional de la República de Colombia, sitio Web del Ministerio TIC: http://goo.gl/bygST, recuperado el 9 de mayo de 2013.

Ministerio TIC (2011b). Estrategia Nacional de Gobierno en Línea, sitio Web del Programa de Gobierno en Línea: http:// goo.gl/8GRd3, recuperado el 9 de mayo de 2013.

Ministerio TIC (2011c). Programa Agenda de Conectividad: Estrategia de Gobierno en Línea. Programa en cifras, sitio Web del Programa de Gobierno en Línea: http://goo.gl/utk1o, recuperado el 9 de mayo de 2013.

Ministerio TIC (2012). Manual 3.1. Implementación de la estrategia de Gobierno en Línea en las entidades del orden nacional en la República de Colombia 2012-2015, del sitio Web del Programa de Gobierno en Línea: http://goo. 
gl//Bpxx, recuperado el 2 de julio de 2013.

Moore, Gordon (1975). Progress in digital integrated electronics. IEEE International Electron Devices Meeting IEDM Technical Digest, United States, pp. 11-13.

Organización de las Naciones Unidas - ONU (2003). Informe de Gobierno Electrónico de la ONU, sitio Web: "United Nation Public Administration Network UNPAN": http://goo.gl/xd1uS, recuperado el 9 de mayo de 2013.

Organización de las Naciones Unidas-ONU (2003-2012). Informes de Gobierno Electrónico de la ONU, sitio Web: "United Nation Public Administration Network - UNPAN": http://goo.gl/ xd1uS, recuperado el 9 de mayo de 2013.

Organización de las Naciones Unidas-ONU (2012). Objetivos de Desarrollo del Milenio - ODM, sitio Web: Portal de los Objetivos de Desarrollo del Milenio ODM: http://goo.gl/BSxuk, recuperado el 4 de mayo de 2013.

Organización para la Cooperación y el Desarrollo Económico-OCDE (2001). Understanding the Digital Divide, Francia, OCDE.

Presidencia de la República (2000). Directiva Presidencial 02 de 2000, recuperado el 4 de mayo de 2013, del sitio Web del programa de Gobierno en Línea: http://goo.gl/FAFh0. Bogotá, Colombia.

Presidencia de la República (2001). Decreto 127 de 2001, recuperado el 4 de mayo de 2013, del sitio Web del programa de Gobierno en Línea: http://goo.gl /iGIRC. Bogotá, Colombia.

Presidencia de la República (2003a). Decreto 3107 de 2003, recuperado el 4 de mayo de 2013, del sitio Web del programa de Gobierno en Línea: http:// goo.gl/N4Xm4. Bogotá, Colombia.
Presidencia de la República (2003b). Decreto 3816 de 2003, recuperado el 4 de mayo de 2013, del sitio Web del programa de Gobierno en Línea: http:// goo.gl/AQqVF. Bogotá, Colombia.

Presidencia de la República (2012). Decreto 19 de 2012, recuperado el 4 de mayo de 2013, del sitio Web del programa de Gobierno en Línea: http://goo. gl/k1YZD. Bogotá, Colombia.

Roberts, Alasdair (2006). Governmantal adaptation to transparency rules, En C. $\mathrm{H}$. Heald, Transparency: The Key to Better Governance? (pp. 107-144). Oxford: Oxford University Press.

Robertson, Scott y Vatrapu, Ravi (2012). Digital government, Annual Review of Information Science and Technology, Vol. 44, No. 1, United States, Association for Information Science y Technology, pp. 317-364.

Srivastava, Shirish y Teo, Thompson (2005). E-government as a guided evolution in Singapore: Vision for the world in the 21st century. Sixty-Fifth Academy of Management Annual Meeting, United States, Academy of Management, pp. 5-10.

Teo, Thompson; Srivastava, Shirish; y Jiang, Li (2008). Trust and electronics government success: An empirical study. Journal of Management Information System, Vol. 28, No. 3, pp.99-131.

Transparencia Internacional-TI (2003-2010). Informes Anuales del Índice de Percepción de Corrupción 2003-2010, sitio Web de Transparencia Internacional: http://goo.gl/ByYzR, recuperado el 4 de mayo de 2013.

United Nations-UN (2014). United Nations EGovernment Survey, sitio Web: http://goo.gl/HEFZpA, recuperado el 6 de febrero de 2015.

Yang, Tung-Mou; Pardo, Theresa y Wu, YiJung (2014). How is information shared across the boundaries of govern- 
Gobierno Electrónico en Colombia: marco normativo y evaluación...

Cardona Madariaga, Diego; Cortés Sánchez, Julián y Ujueta, Sandra

ment agencies? An e-Government case study, Government Information Quaterly, Vol. 31, No. 4, United States, pp. 637-652.
Zheng, Yueping; Lauer, Hindy y Holzer, Marc (2014). The impact of government form on e-Participation: A study of New Jersey municipalities. Government Information Quarterly, Vol. 31, No. 4, United States, pp. 663-699. 